\title{
CIRCULARLY POLARIZED LIGHT-INDUCED AXIAL ANISOTROPY IN ACHIRAL ISOTROPIC MEDIA. MAGNETIC DIPOLE INTERACTION WITH RADIATION
}

\author{
S. WOŹNIAK \\ Nonlinear Optics Division, Institute of Physics, A. Mickiewicz University \\ Grunwaldzka 6, 60-780 Poznań, Poland
}

(Reccived February 10,1994)

\begin{abstract}
The analytical explanation of axial birefringence revealed earlicr by the technique of field applicd molecular dynamics computer simulation is given. The point group symmetries of achiral molecules admitting the effect are determined and the estimation of the anisotropy is presented. The effect may give information on off-diagonal elements of the Rosenfeld polarizability tensor of achiral molecules.
\end{abstract}

PACS numbers: 12.25.Lc, 42.65.An

\section{Introduction}

Various optical birefringences can be induced in isotropic media by a static electric or magnetic field, among them there is the Kerr effect and the CottonMouton effect [1-5], the Faraday effect $[6,7]$ and magnetochiral birefringence [8-13]. The birefringence can also be induced by these two static fields applied simultaneously [14-18], an clectric ficld gradient [19] or by a high intensity optical beam [3, 20-25].

Recently [26] the technique of field applied molecular dynamics (FMD) computer simulation has been used to show the existence of a novel anisotropy induced by circularly polarized (c.p.) light in media composed of chiral or achiral molecules. In that paper two real torques acting on a molecule in the presence of c.p. light were taken into consideration:

$$
\begin{aligned}
& T_{1}=\frac{1}{2} \mu \times E^{*}+\text { c.c. } \\
& T_{2}=\frac{1}{2} m \times B^{*}+\text { c.c. },
\end{aligned}
$$

where $\boldsymbol{\mu}$ is the electric dipole moment induced in a molecule by the magnetic field $\boldsymbol{B}$ of the c.p. light and $\boldsymbol{m}$ is the magnetic dipole moment induced by the electric field $\boldsymbol{E}$ of the c.p. light ( $\boldsymbol{E}^{*}$ and $\boldsymbol{B}^{*}$ denote the complex conjugates of $\boldsymbol{E}$ and $\boldsymbol{B}$ ). 
The torques (1) and (2) lead to the light intensity dependent axial birefringence for chiral as well as achiral molecules possessing appropriate symmetries, as was shown by the FMD technique for (S)-CIIBrClF and $\mathrm{II}_{2} \mathrm{O}$, respectively [26].

Additional contributions to the effect due to electric quadrupole interaction with electromagnetic radiation arc in gencral comparable with the magnetic dipole contributions and they will be presented in a separate paper.

\section{The basic formulae}

We consider an isotropic molecular system in which a monochromatic light wave with the electric ficld

$$
\boldsymbol{E}(r, t)=\frac{1}{2} \boldsymbol{E}(\omega, \boldsymbol{k}) \exp \left[-\mathrm{i} \omega\left(l-\frac{n}{c} \boldsymbol{r} \cdot \boldsymbol{s}\right)\right]+\text { c.c. }
$$

propagates in the $s$-direction, $n$ is the light refractive index, $k$ - the propagation vector of the wave, and $c$ - the velocity of light in vacuum.

The optical properties of liquid and gascous media composed of non-interacting molecules are described by the material equations for the elcctric $\mathcal{D}$ and magnctic $\mathcal{B}$ flux density:

$$
\begin{aligned}
& \mathcal{D}(\omega, k, \boldsymbol{E}, \boldsymbol{B})=\varepsilon_{0} \boldsymbol{E}(\omega, k)+N\langle\boldsymbol{\mu}(\omega, k, \boldsymbol{E}, \boldsymbol{B})\rangle_{E, B}, \\
& \mathcal{B}(\omega, k, \boldsymbol{E}, \boldsymbol{B})=\mu_{0} \boldsymbol{I}(\omega, k)+\mu_{0} N\langle\boldsymbol{m}(\omega, k, \boldsymbol{E}, \boldsymbol{B})\rangle_{E, B},
\end{aligned}
$$

where $\boldsymbol{\mu}(\omega, k, \boldsymbol{E}, \boldsymbol{B})$ and $\boldsymbol{m}(\omega, \boldsymbol{k}, \boldsymbol{E}, \boldsymbol{B})$ are the clectric dipolc and magnetic dipole moment, respectively, induced in the moleculc by the fields $\boldsymbol{E}$ and $\boldsymbol{B}$ of the light, $N$ is the number of molecules per unit volume, $\varepsilon_{0}$ and $\mu_{0}$ - the electric and magnetic pormittivity of vacuum, and the symbol $\langle\ldots\rangle_{E, B}$ stands for the space average in the presence of the fields $\boldsymbol{E}$ and $\boldsymbol{B} ; \boldsymbol{H}(\omega, k)=\mu_{0}^{-1} \boldsymbol{B}(\omega, k)$ is the magnetic field of the lighte.

Since we are interested in the analytical explanation of the optical anisotropy of the medium due to the torques (1) and (2), we will take now into consideration only optically induced reorientational processes giving any contribution to the refractive index. The induced dipole momonts $\mu$ and $m$ oscillating with the frequcncy $\omega$ can be written in the following approximation:

$$
\begin{aligned}
& \mu_{i}(\omega, \boldsymbol{E}, \boldsymbol{B})={ }^{\mathrm{e}} \alpha_{i j}^{\mathrm{e}}(-\omega ; \omega) E_{j}+{ }^{\mathrm{e}} \alpha_{i j}^{\mathrm{m}}(-\omega ; \omega) B_{j}, \\
& m_{i}(\omega, \boldsymbol{E}, \boldsymbol{B})={ }^{\mathrm{m}} \alpha_{i j}^{\mathrm{e}}(-\omega ; \omega) E_{j},
\end{aligned}
$$

where ${ }^{\mathrm{e}} \boldsymbol{\alpha}^{\mathrm{e}}(-\omega ; \omega)$ and ${ }^{\mathrm{e}} \boldsymbol{\alpha}^{\mathrm{m}}(-\omega ; \omega)$ is the elcctric dipole polarizability related to dipolar-electric and dipolar-magnetic transitions, respectively, ${ }^{\mathrm{m}} \boldsymbol{\alpha}^{\mathrm{e}}(-\omega ; \omega)$ is the magnetic dipole polarizability related to dipolar-electric transitions (the electric quadrupole interaction with radiation is not considered here and it will be presented in a separate paper).

The space average in the presence of the electromagnetic ficld can be carried out using the formula [3]:

$$
\langle\Phi\rangle_{E, B}=\langle\Phi\rangle-\frac{1}{k_{\mathrm{B}} T}(\langle\Phi U\rangle-\langle\Phi\rangle\langle U\rangle)+\ldots
$$


where $k_{\mathrm{B}}$ is the Boltzmann constant, $T$ - the temperature, and $U$ - the potential energy of the molecule in the presence of the laser beam:

$$
\begin{aligned}
U= & -\frac{1}{2}{ }^{\mathrm{e}} \alpha_{i j}^{\mathrm{e}}(-\omega ; \omega) E_{i}^{*} E_{j}-\frac{1}{2}{ }^{\mathrm{e}} \alpha_{i j}^{\mathrm{m}}(-\omega ; \omega) E_{i}^{*} B_{j} \\
& -\frac{1}{2}{ }^{\mathrm{m}} \alpha_{i j}^{\mathrm{e}}(-\omega ; \omega) B_{i}^{*} E_{j}+\text { c.c. }
\end{aligned}
$$

In general, for molccules with complex wave functions, the polarizabilities ${ }^{\mathrm{e}} \boldsymbol{\alpha}^{\mathrm{e}},{ }^{\mathrm{e}} \boldsymbol{\alpha}^{\mathrm{m}}$ and ${ }^{\mathrm{m}} \boldsymbol{\alpha}^{\mathrm{e}}$ can be represented as follows (from liere on we will not indicate anymore the frequency in the symbols of the polarizabilities, writing $\alpha$ instead of $\alpha(-\omega ; \omega))$ :

$$
\begin{aligned}
& { }^{\mathrm{e}} \alpha_{i j}^{\mathrm{e}}={ }^{\mathrm{e}} \beta_{i j}^{\mathrm{e}}+\mathrm{i}{ }^{\mathrm{e}} \gamma_{i j}^{\mathrm{e}}={ }^{\mathrm{e}} \beta_{j i}^{\mathrm{e}}-\mathrm{i}^{\mathrm{e}} \gamma_{j i}^{\mathrm{e}}, \\
& { }^{\mathrm{e}} \alpha_{i j}^{\mathrm{m}}={ }^{\mathrm{e}} \beta_{i j}^{\mathrm{m}}+\mathrm{i}^{\mathrm{e}} \gamma_{i j}^{\mathrm{m}}, \\
& { }^{\mathrm{m}} \alpha_{i j}^{\mathrm{e}}={ }^{\mathrm{m}} \beta_{i j}^{\mathrm{e}}+\mathrm{i}^{\mathrm{m}} \gamma_{i j}^{\mathrm{e}}={ }^{\mathrm{e}} \beta_{j i}^{\mathrm{m}}-\mathrm{i}{ }^{\mathrm{e}} \gamma_{j i}^{\mathrm{m}},
\end{aligned}
$$

where the tensor components ${ }^{\mathrm{e}} \beta_{i j}^{\mathrm{e}},{ }^{\mathrm{e}} \gamma_{i j}^{\mathrm{e}},{ }^{\mathrm{e}} \beta_{i j}^{\mathrm{m}},{ }^{\mathrm{e}} \gamma_{i j}^{\mathrm{m}},{ }^{\mathrm{m}} \beta_{i j}^{\mathrm{e}}$ and ${ }^{\mathrm{m}} \gamma_{i j}^{\mathrm{e}}$ are real in the absence of absorption with the quantum mechanical forms given in Ref. [8]. For molecules with real wave functions the tensor components ${ }^{\mathrm{e}} \gamma_{i j}^{\mathrm{e}},{ }^{\mathrm{e}} \beta_{i j}^{\mathrm{m}}$ and ${ }^{\mathrm{m}} \beta_{i j}^{\mathrm{e}}$ are equal to zero $[8,27,28]$.

On transforming the material Eqs. (4) and (5) to circular basis and on solving them in this basis jointly with the Maxwell equations we obtain expressions defining the refractive indices $n_{+}$and $n_{-}$for right and left c.p. light, respectively, propagating in the $z$-direction of the laboratory frame of coordinates $\{X, Y, Z\}$ in a medium composed of achiral molecules

$$
n_{ \pm}=n_{0}+n_{T}^{\mathrm{NL}}|\boldsymbol{E}|^{2} \pm F_{T} \mathrm{i}\left(\boldsymbol{E} \times \boldsymbol{E}^{*}\right)_{z} \pm D_{T} \mathrm{i}\left(\boldsymbol{E}^{*} \cdot \boldsymbol{B}\right)+M_{T}\left(\boldsymbol{E}^{*} \times \boldsymbol{B}\right)_{z}
$$

where

$$
n_{0}=\left(1+\frac{N}{\varepsilon_{0}} \mathrm{e} \beta^{\mathrm{c}}\right)^{1 / 2}
$$

is the refractive index for linearly polarized light with ${ }^{\mathrm{e}} \beta^{\mathrm{e}}=\frac{1}{3}{ }^{\mathrm{e}} \beta_{\alpha \alpha}^{\mathrm{e}}$ being the mcan value of the polarizability ${ }^{\mathrm{e}} \boldsymbol{\beta}^{\mathrm{e}}(-\omega ; \omega)$

$$
n_{T}^{\mathrm{NL}}=\frac{N}{180 \varepsilon_{0} n_{0} k_{\mathrm{B}} T}\left(3^{\mathrm{e}} \beta_{\alpha \beta}^{\mathrm{e}}{ }^{\mathrm{e}} \beta_{\alpha \beta}^{\mathrm{e}}-{ }^{\mathrm{e}} \beta_{\alpha \alpha}^{\mathrm{e}}{ }^{\mathrm{e}} \beta_{\beta \beta}^{\mathrm{e}}\right)
$$

describes the light intensity-dependent change of the refractive index, and the other constants have the following form:

$$
\begin{aligned}
F_{T} & =\frac{N}{12 \varepsilon_{0} n_{0} k_{\mathrm{B}} T}{ }^{\mathrm{e}} \gamma_{\alpha \beta}^{\mathrm{e}}{ }^{\mathrm{e}} \gamma_{\alpha \beta}^{\mathrm{e}}, \\
D_{T} & =\frac{N}{30 \varepsilon_{0} c k_{\mathrm{B}} T}\left({ }^{\mathrm{e}} \gamma_{\alpha \beta}^{\mathrm{m}}{ }^{\mathrm{e}} \gamma_{\alpha \beta}^{\mathrm{m}}+{ }^{\mathrm{e}} \gamma_{\alpha \beta}^{\mathrm{m}}{ }^{\mathrm{e}} \gamma_{\beta \alpha}^{\mathrm{m}}\right), \\
M_{T} & =\frac{N}{6 \varepsilon_{0} c k_{\mathrm{B}} T}\left({ }^{\mathrm{e}} \beta_{\alpha \beta}^{\mathrm{m}}{ }^{\mathrm{e}} \beta_{\alpha \beta}^{\mathrm{m}}-{ }^{\mathrm{e}} \beta_{\alpha \beta}^{\mathrm{m}}{ }^{\mathrm{e}} \beta_{\beta \alpha}^{\mathrm{m}}\right),
\end{aligned}
$$


and they are related to optically induced circular and axial birefringence discussed with details in the separate paper [29]; $\left(\boldsymbol{E} \times \boldsymbol{E}^{*}\right)_{z}$ and $\left(\boldsymbol{E}^{*} \times \boldsymbol{B}\right)_{z}$ in Eq. (13) denote the $z$-component of the vector product in the brackets. The temperature dependent parameters $F_{T}$ and $M_{T}$ disappear if the ground state of the molecule is non-degenerate.

On the other hand the refractive index of the linearly polarized light with the same intensity is

$$
n=n_{0}+n_{T}^{\mathrm{NL}}|E|^{2}+M_{T}\left(E^{*} \times \boldsymbol{B}\right)_{z} .
$$

For right (R) and left (L) c.p. light propagating in $z$-direction we have [29]:

$$
\begin{aligned}
& \mathrm{i}\left(\boldsymbol{E} \times \boldsymbol{E}^{*}\right)_{z}^{\mathrm{L}}=-\mathrm{i}\left(\boldsymbol{E} \times \boldsymbol{E}^{*}\right)_{z}^{\mathrm{R}}=E^{2}, \\
& \mathrm{i}\left(\boldsymbol{E}^{*} \cdot \boldsymbol{B}\right)^{\mathrm{L}}=-\mathrm{i}\left(\boldsymbol{E}^{*} \cdot \boldsymbol{B}\right)^{\mathrm{R}}=E B, \\
& \left(\boldsymbol{E}^{*} \times \boldsymbol{B}\right)_{z}^{\mathrm{L}}=\left(\boldsymbol{E}^{*} \times \boldsymbol{B}\right)_{z}^{\mathrm{R}}=E B,
\end{aligned}
$$

and, moreover, for linearly polarized light

$$
\left(\boldsymbol{E}^{*} \times \boldsymbol{B}\right)_{z}=E B .
$$

The effect, which we are interested in, is described by the difference $\Delta n=n-n_{ \pm}$ of the refractive indices for linearly and circularly polarized light

$$
\Delta n=n-n_{ \pm}=F_{T} E^{2}+D_{T} E B .
$$

In general case the nonlinear terms describing nonlinear electronic distortion should be included in Eqs. (13), (19) and (24) (see Sec. 3).

\section{Discussion}

For achiral molecules with non-degenerate electronic ground state the constant $F_{T}=0$ and the effect depends on components of the polarizability tensor ${ }^{\mathrm{e}} \gamma^{\mathrm{m}}$ containing one electric dipole and one magnetic dipole transition (the Rosenfeld polarizability) as wc could expect from the torques given by Eqs. (1) and (2) (see also Eqs. (20) and (21) in Ref. [26]: the effect revealed by the FMD technique corresponds to the effect described in the present paper by the term $D_{T} E B$ ). The non-zero components of the tensor ${ }^{\mathrm{e}} \gamma_{\alpha \beta}^{\mathrm{m}}$ for all point symmetries of achiral molecules are presented in Table. From Table we have

$$
D_{T}=\frac{N}{30 \varepsilon_{0} c k_{\mathrm{B}} T}\left[\left({ }^{\mathrm{e}} \gamma_{13}^{\mathrm{m}}+{ }^{\mathrm{e}} \gamma_{31}^{\mathrm{m}}\right)^{2}+\left({ }^{\mathrm{e}} \gamma_{23}^{\mathrm{m}}+{ }^{\mathrm{e}} \gamma_{32}^{\mathrm{m}}\right)^{2}\right]
$$

for molecules with the point group $m\left(C_{1 h}\right)$;

$$
D_{T}=\frac{N}{30 \varepsilon_{0} c k_{\mathrm{B}} T}\left({ }^{\mathrm{e}} \gamma_{12}^{\mathrm{m}}+{ }^{\mathrm{e}} \gamma_{21}^{\mathrm{m}}\right)^{2}
$$

for molecules having the symmetry $m m 2\left(C_{2 v}\right)$;

$$
D_{T}=\frac{2 N}{15 \varepsilon_{0} c k_{\mathrm{B}} T}\left({ }^{\mathrm{e}} \gamma_{12}^{\mathrm{m}}\right)^{2}
$$

for molecules with the symmetry $\overline{4}\left(S_{4}\right)$. For the remaining point group symmetries of achiral molecules the constant $D_{T}$ is equal to zero. 
TABLE

The rank-2 axial tensor e $\gamma_{\alpha \beta}^{\mathrm{m}}$ for all point group symmetries of achiral molecules [27]. The point groups are given in the international notation, in brackets the Schoenflies notation.

\begin{tabular}{l|l}
\hline \multicolumn{1}{c|}{ Point group } & \multicolumn{1}{c}{${ }^{\mathrm{e}} \gamma_{\alpha \beta}^{\mathrm{m}}$} \\
\hline$m\left(C_{s}\right)$ & ${ }^{\mathrm{e}} \gamma_{13}^{\mathrm{m}},{ }^{\mathrm{e}} \gamma_{31}^{\mathrm{m}},{ }^{\mathrm{e}} \gamma_{23}^{\mathrm{m}},{ }^{\mathrm{e}} \gamma_{32}^{\mathrm{m}}$ \\
$m m 2\left(C_{2 v}\right)$ & $\mathrm{e} \gamma_{12}^{\mathrm{m}},{ }^{\mathrm{e}} \gamma_{21}^{\mathrm{m}}$ \\
$\overline{4}\left(S_{4}\right)$ & ${ }^{\mathrm{e}} \gamma_{11}^{\mathrm{m}}=-{ }^{\mathrm{e}} \gamma_{22}^{\mathrm{m}},{ }^{\mathrm{e}} \gamma_{12}^{\mathrm{m}}={ }^{\mathrm{e}} \gamma_{21}^{\mathrm{m}}$ \\
$3 m\left(C_{3 v}\right), 4 m m\left(C_{4 v}\right), 6 m m\left(C_{6 v}\right), \infty m\left(C_{\infty v}\right)$ & ${ }^{\mathrm{e}} \gamma_{12}^{\mathrm{m}}=-{ }^{\mathrm{e}} \gamma_{21}^{\mathrm{m}}$ \\
$\overline{4} 2 m\left(D_{2 d}\right)$ & ${ }^{\mathrm{e}} \gamma_{11}^{\mathrm{m}}=-{ }^{\mathrm{e}} \gamma_{22}^{\mathrm{m}}$ \\
\hline
\end{tabular}

For the remaining point groups of achiral molecules all components vanish

The above results show that the effect revealed by the FMD technique and discussed in this paper may give information on off-diagonal elements of the Rosenfeld polarizability tensor, also for non-dipolar molecules having the symmetry of the point group $\overline{4}\left(S_{4}\right)$, when there is no contribution of the components ${ }^{\mathrm{e}} \gamma_{\alpha \beta}^{\mathrm{m}}$ to the linear [30] and nonlinear [31] electric Rayleigh optical activity.

As a rough estimate we take ${ }^{\mathrm{e}} \gamma_{12}^{\mathrm{m}} \approx 10^{-34} A^{2} J^{-1} \mathrm{~m}^{3} s$ (in the paper [30] an experimental value for ${ }^{\mathrm{e}} \gamma_{12}^{\mathrm{m}}$ for the molecule $\mathrm{CH}_{3} \mathrm{Cl}$ of $-0.34 \times 10^{-34} \mathrm{~A}^{2} \mathrm{~J}^{-1} \mathrm{~m}^{3} \mathrm{~s}$ is given from linear electric Rayleigh optical activity measurements) and $N \approx$ $10^{28} \mathrm{~m}^{-3}$ which give

$$
\Delta n=D_{T} E B \approx 10^{-26} E^{2}
$$

with $E$ in $\mathrm{V} \mathrm{m}^{-1}$. In this case the effect secms to be too small for measurement (it could be measured by focusing a giant ruby laser pulse producing a field $10^{9}-10^{10} \mathrm{~V} / \mathrm{m}$ but the optical breakdown may appear). We hope that for some achiral molecules (macromolecules) with larger off-diagonal components of the Rosenfeld polarizability the effect may be observable in much lower optical field.

For achiral molecules with degenerate electronic ground state the main contribution to the difference $\Delta n$ given by Eq. (24) may come from the first term $F_{T} E^{2}$. Taking e $\gamma^{\mathrm{e}} \approx 1.7 \times 10^{-40} C^{2} m^{2} J^{-1}$ [24] we have at room temperature

$\Delta n \approx 1.5 \times 10^{-21} E^{2}$,

where $E$ is in SI units. The effect might be detectable even for the field $E \approx$ $10^{7} \mathrm{~V} / \mathrm{m}$ which corresponds to the light intensity of $10^{11} \mathrm{~W} / \mathrm{m}^{2}$.

We would like to notice that, in general, when the nonlinear electronic distortion is taken into consideration, the expressions for the indices $n_{ \pm}, n$ and $\Delta n$ have the form of Eqs. (13), (19) and (24) with the constants $n^{\mathrm{NL}}=n_{0}^{\mathrm{NL}}+n_{T}^{\mathrm{NL}}$, $F=F_{0}+F_{T}, D=D_{0}+D_{T}$ and $M=M_{0}+M_{T}$ instead of $n_{T}^{\mathrm{NL}}, F_{T}, D_{T}$ and $M_{T}$, and the new constants contain, besides the temperature-dependent terms, also the temperature-independent terms (denoted by the subscripts " 0 ") related to nonlinear interaction of molecules with the radiation [29]. For anisotropic molecules the temperature-dependent terms (if they are not equal to zero because of molecular 
symmetry) are usually much larger than the respective terms describing the nonlinear electronic distortion $[24,29]$ and in such case the tempcraturc-independent terms may be neglected. Since, moreover, $F_{0} E^{2} \gg D_{0} E B$ [29] we have for molecules with non-degenerate electronic ground state

$$
\Delta n=n-n_{ \pm}=F_{0} E^{2}+D_{T} E B,
$$

with

$$
F_{0}=\frac{N}{16 \varepsilon_{0} n_{0}}\left[{ }^{\mathrm{e}} \beta_{\alpha \beta \beta \alpha}^{\mathrm{eee}}(-\omega ; \omega,-\omega, \omega)-{ }^{\mathrm{e}} \beta_{\alpha \beta \alpha \beta}^{\mathrm{eee}}(-\omega ; \omega,-\omega, \omega)\right],
$$

which is non-zero for all atoms and molccules. The quantum mechanical form of the hyperpolarizability ${ }^{\mathrm{e}} \beta_{\alpha \beta \gamma \delta}^{\mathrm{eee}}(-\omega ; \omega,-\omega, \omega)$ is given in Ref. [29].

As a rough estimate of the constant $F_{0}$ we take e ${ }^{\text {eee }} \approx 10^{-60} \mathrm{C}^{4} \mathrm{~m}^{4} \mathrm{~J}^{-3}$ which is typical for such molecules as $\mathrm{C}_{2} \mathrm{II}_{6}, \mathrm{C}_{6} \mathrm{I}_{6}, \mathrm{CS}_{2}[5]$ and which leads to $F_{0} \approx 10^{-22} \mathrm{~m}^{2} \mathrm{~V}^{-2}$. The above estimation means that the contribution of the term $F_{0} E^{2}$ to the effect discussed in this paper may be observable for c.p. light with the amplitude of the clectric field $E \approx 10^{8} \mathrm{~V} / \mathrm{m}$.

\section{Acknowledgment}

The author wishes to thank Professor G. Wagnière for his helpful discussions and remarks throughout the presented investigation.

\section{References}

[1] M. Born, Optik, Springer, Berlin 1933.

[2] M. Volkensteyn, Molekularnaya Optika, Gostekizdat, Moscow 1951.

[3] S. Kielich, Nonlincar Molecular Optics, Nauka, Moscow 1981.

[4] S. Kïlich, Acta Phys. Pol. 22, 65, 299 (1962).

[5] S. Kiclich, in: Dielectric and Related Molecular Processes, Ed. M. Davies, Chemical Society of London, London 1972.

[6] M.P. Groenewege, Mol. Phys. 5, 541 (1962).

[7] A.D. Buckingham, P.J. Stephens, Ann. Rev. Phys. Chem. 17, 38 (1966).

[8] S. Woźniak, R. Zawodny, Acla Phys. Pol. A 61, 175 (1982); 68, 675 (1985).

[9] G. Wagnière, A. Meier, Chem. Phys. Lell. 93, 78 (1982).

[10] L.D. Barron, J. Vrbancich, Mol. Phys. 51, 715 (1984).

[11] S. Woźniak, Mol. Phys. 59, 421 (1986); Phys. Lett. 119, 256 (1986).

[12] S. Woźniak, J. Chem. Phys. 85, 4217 (1986).

[13] S. Woźniak, Acta Phys. Pol. A 72, 779 (1987).

[14] S. Kielich, Acta Phys. Pol. 31, 929 (1967).

[15] N.B. Baranova, Yu.V. Bogdanov, B.Ya. Zel'dovich, Optics Commun. 22, 243 (1977).

[16] A.D. Buckingham, R.A. Shatwell, Chem. Phys. 35, 353 (1978).

[17] E.B. Graham, R.E. Raab, Mol. Phys. 52, 1241 (1984).

[18] E.B. Graham, R.E. Raab, Proc. R. Soc. Lond. A 390, 73 (1983). 
[19] D.A. Imrie, R.E. Raab, Mol. Phys. 74, 833 (1991).

[20] A.D. Buckingham, Proc. Phys. Soc. B 69, 344 (1956).

[21] G. Mayer, F. Gires, Compt. Rend. Acad. Sci. 258, 2039 (1964).

[22] S. Kielich, J.R. Lalanne, F.B. Martin, J. Phys. (Paris) 33, 191 (1972).

[23] K. Sala, M.C. Richardson, Phys. Rev. A 12, 1036 (1975).

[24] P.W. Atkins, M.H. Miller, Mol. Phys. 15, 503 (1968).

[25] M.W. Evans, S. Woźniak, G. Wagnière, Physica B 175, 412 (1991).

[26] M.W. Evans, S. Wożniak, G. Wagnière, Physica B 170, 133 (1992).

[27] S. Woźniak, R. Zawodny, Chem. Phys. 103, 303 (1986).

[28] A.D. Buckingham, R.E. Raab, Proc. R. Soc. Lond. 345, 365 (1975).

[29] S. Woźniak, Mol. Phys. 80, 355 (1993).

[30] A.D. Buckingham, R.A. Shatwell, Phys. Rev. Lelt. 45, 21 (1980).

[31] S. Wó́niak, Phys. Lett. A 148, 188 (1990). 